\title{
Meta-analysis
}

\section{A meta-analysis of case-reports and case-series of dacrystic epilepsy in children and adolescence}

\author{
Chandan Raybarman*
}

Department of Paediatrics, Gauri Devi Institute of Medical Sciences and Hospital, Durgapur, West Bengal, India

Received: 27 August 2021

Revised: 24 September 2021

Accepted: 07 October 2021

\author{
*Correspondence: \\ Dr. Chandan Raybarman, \\ E-mail: raybarmanchandan@yahoo.com
}

Copyright: ( ) the author(s), publisher and licensee Medip Academy. This is an open-access article distributed under the terms of the Creative Commons Attribution Non-Commercial License, which permits unrestricted non-commercial use, distribution, and reproduction in any medium, provided the original work is properly cited.

\begin{abstract}
This meta-analysis aimed to advance our knowledge about dacrystic epilepsy in children in the present time. PubMed searches for peer-reviewed case reports and case series were conducted using the keywords "dacrystic epilepsy", "dacrystic seizures", "crying epilepsy", "ictal crying", "crying seizures". The databases were developed in accordance with preferred reporting items for systematic reviews and meta-analyses (PRISMA) guidelines. The author collected relevant information to characterise the study population including clinical outcome. Eleven studies out of 353 citations between 1998 and 26 May 2021 met the inclusion criteria, including both single cases and series pertaining to dacrystic epilepsy. Eight case reports and three case series were eligible for this meta-analysis and included twenty one cases. The seizure patterns were dacrystic seizures alone in seven cases (33\%), and a combination of dacrystic seizures and gelastic seizures in fourteen cases $(67 \%)$. Neuroimaging revealed structural abnormality in $95 \%$ cases. Hypothalamic hamartoma was found in most of the cases (79\%) with combined dacrystic seizures and gelastic seizures, whereas it was found in one case (16\%) with dacrystic seizures alone. The other underlying lesions in children with dacrystic seizures alone were subependymal nodules and cortical hamartomas (17\%), left mesial temporal sclerosis (33\%), and cortical dysplasia (17\%). Regarding outcome, antiepileptic drugs alone achieved seizure freedom in four cases (22\%) only and others $(78 \%)$ were difficult to treat cases excluding three cases where treatments were not mentioned. Six cases underwent surgical intervention and two cases received ablative radiotherapy. Lesional dacrystic epilepsy is predominant and pharmaco-resistant in children. However, antiepileptic drugs lead to achieving seizure remission in few cases.
\end{abstract}

Keywords: Meta-analysis, Dacrystic, Gelastic, Seizures, Hypothalamic hamartoma

\section{INTRODUCTION}

One of the expressions of an emotion is crying in a context of sadness. This subjective feeling is unique of human behaviour. In fact this emotional act is dependent partly upon the cognitive appraisal of the emotional triggering stimuli. In this regard, happiness begets laughter another emotional expression of human behaviour. However, happiness can be an emotional triggering stimulus of a distinctive pattern of crying. Therefore, a hybridisation of laughter and crying is existing as a distinctive pattern. ${ }^{1}$ However, crying may be pathological and can be expressed without subjective feeling and emotional stimuli. This inappropriate emotional display cannot voluntarily prevent from happening or stop once it has occurred. Symptoms besides crying spell may present such as lacrimation, grimacing, sobbing, sad facial expression, or yelling.

Considering the disassociation between emotional experience and expression it is very difficult to distinguish the essence of pathological crying which is more or less identical to the normal condition. In this regard, the phenomena of laughing and crying are complex in the 
perspective of neuroanatomical and functional framework. $^{2}$

A unique clinical presentation of an epileptic seizure is episodic crying with preserved consciousness. This is a rare presentation of an epileptic seizure occurring with an estimated frequency of $0.1 \%$ to $0.13 \%$ in patients admitted for long-term video monitoring and rarely occurs as an isolated clinical feature. ${ }^{3,4}$ The first description of crying seizures dates back to 1906 when Zilgien described "spasmodic crying" in a patient who finally died of luetic pachymeningitis. ${ }^{5}$ Dacrystic epilepsy was first named by Offen and colleagues in 1976 in his case report of a 69 year old male with crying seizures. ${ }^{6}$ Since then, dacrystic seizures (DS) have been studied and reported infrequently in children. In true sense, dacrystic episodes of crying manifest without sadness. Some patients manifest both gelastic episodes of mirthless laughter and dacrystic episodes of crying without sadness.

Dacrystic epilepsy exists as a distinct subgroup of focal emotional seizures category and share partly in global burden of epilepsy. ${ }^{7}$ The symptom "crying", is nonspecific and its stereotypical ictal nature can be difficult to ascertain in many cases. Luciano et al suggests that dacrystic epilepsy may be more common than reported in the literature. ${ }^{8}$ Truely speaking there is hardly found any study accumulating all the cases reported with dacrystic epilepsy in children.

Considering challenges for meta-analyses in small populations and rare diseases the present study is designed to advance our knowledge about dacrystic epilepsy in children in the present time.

\section{METHODS}

The present study was designed as a meta-analysis to characterise dacrystic epilepsy in children and to describe the clinical presentation of DS in relationship to the underlying aetiology. The study was done in the department of paediatrics of Gauri Devi Institute of Medical Sciences and Hospital, West Bengal, India.

\section{Search strategy}

A systematic search of the PubMed database was made in order to identify all of the articles containing the words " dacrystic epilepsy", "dacrystic seizures", "crying epilepsy”, "ictal crying”, "crying seizures". Language was restricted to English. The search was conducted on 25 May 2021. A thorough manual review of case-reports and case series was performed using cross-references from identified original articles.

\section{Selection criteria}

Studies meeting the following criteria were considered for inclusion: diagnosis of dacrystic epilepsy in the setting of convincing clinical evidence; patients with DS only; patients with a combination of gelastic seizures (GS) and DS; and age at onset of seizures 18 years or less. We included only case reports and case series retrieved in full text. Considering the all inclusion criteria, letters to the editor were also included. A two-stage study selection process was used: all titles and abstracts were initially screened for potential relevance, with full texts of the potentially relevant references being screened subsequently. The exclusion criteria were patients who do not neatly fit into recognized dacrystic epilepsy and studies that publish in other than English language.

\section{Data extraction}

The author independently performed the literature search and screened all study titles and abstracts. From the eligible articles, full-text versions were retrieved and reviewed. Data were extracted and checked.

The author collected information to develop the study population about group sizes, sex distribution, age at data acquisition, age at epilepsy onset, duration of epilepsy, antiepileptic drug usage and presumed aetiology, seizures semiology, electroencephalography (EEG) and magnetic resonance imaging (MRI) findings, treatment, and clinical outcome in terms of seizure control.

Case report and case series databases were developed in accordance with the preferred reporting items for systematic reviews and meta-analyses (PRISMA) guidelines. ${ }^{9}$

Descriptive statistics were used to demonstrate baseline characteristics of the patients expressed as means \pm standard deviations. The online quick standard deviation and variance calculator (https://www.socscistatistics.com) was used to analyse the data. Categorical variables are expressed as numbers and percentages.

\section{RESULTS}

Database searches through 26 May 2021 identified 353 citations with publication dates between 1998 and 26 May 2021, of which 109 duplicates removed, leaving 244 reports for screening. After screening, 38 articles were left for eligibility assessment. We excluded 6 articles not in English and two articles could not be retrieved. Finally 30 articles were left for eligibility assessment of which 11 articles met inclusion criteria in accordance with PRISMA guidelines (Figure 1). ${ }^{9}$ The case reports were aggregated quantitatively, rather than analysing them qualitatively. These 11 articles reported a total of 21 cases. Out of 11 articles, 8 articles presented a single patient case report of which 2 articles were letters to the editor fulfilling the inclusion criteria, and 3 articles presented case series of more than one patient. The median number of patients in articles with 'case series' in the title was found to be four (range 2-7). It was observed also that the chronology of 
published case reports and case series from year to year was not steady (Figure 2). 3,4,10-18

The study population was comprised of 21 patients of which DS alone cases were $7(33 \%)$ and a combination of DS and GS were 14 (67\%) in number (Table 1). The mean age was 6.25 6.59 years (range, 0.1-19 years) at data acquisition. Categorically it was $8.2 \pm 7.79$ years (range, 0.6-19 years) in DS alone group and 5.28 \pm 5.98 years (range, 0.1-19 years) in combined DS and GS group. The mean age of seizure onset was $3.2 \pm 3.26$ years (range, 0.02 10 years). Categorically it was $3.1 \pm 3.16$ years (range, $0.4-$ 9 years) in DS alone group and $3.25 \pm 3.43$ years (range, $0.02-10$ years) in combined DS and GS group.

The study population was $52.4 \%$ male (11 patients) and $47.6 \%$ female (10 patients). Categorically there was very negligible variation in this proportion (Table 1).

The distribution of EEG results were depicted in the pie chart (Figure 3d). EEG results were retrieved in all cases of which abnormality was found in $67 \%$. Categorically $19 \%$ of cases had right hemispheric focal epileptiform discharges and $14 \%$ of cases had left hemispheric focal epileptiform discharges. In contrast the EEG showed bilateral hemispheric epileptiform discharges in 33\% of cases. Normal routine EEG was found in $34 \%$ of cases in the setting of convincing clinical evidence of DS.

The basic findings of the study cohort including the treatment outcome were depicted in Table 2. Brain imaging (MRI or CT) was done in $95 \%$ of cases in which structural abnormality was found in all cases (95\%) except one $(5 \%)$. Only one case $(4.76 \%)$ did not undergo neuroimaging. The structural aetiology of the patients with DS is shown categorically in the pie chart (Figure 3). The accumulated data showed that most of the cases $(60 \%)$ were associated with hypothalamic hematomas (HH) (Figure 3a). Categorically $79 \%$ of cases with a combination of DS and GS were associated with $\mathrm{HH}$ (Figure $3 \mathrm{~b}$ ). In contrast, $16 \%$ of cases of DS without GS were associated with $\mathrm{HH}$ (Figure $3 \mathrm{c}$ ). The other structural abnormalities like bilateral temporoparietal gliosis (7\%), cortical dysplasia (7\%), and acquired cerebellar atrophy (7\%) were equally distributed in cases with combined DS and GS. Comparatively mesial temporal sclerosis (33\%), cortical dysplasia (17\%), sub-ependymal nodule and cortical hamartoma (17\%) were found in cases with DS alone. Normal neuroimaging was depicted in $17 \%$ cases with DS alone.

Cumulatively $86 \%$ of patients received antiepileptic drugs (AEDs) except for three cases (14\%) where AED treatment was not mentioned. Regarding outcome, antiepileptic drugs alone achieved seizure freedom in four cases $(22 \%)$ and others $(78 \%)$ were difficult to treat cases excluding three cases where treatments were not mentioned.

The dosage patterns to which patients were exposed daily were mentioned only in two case reports. These were vigabatrin (750 mg daily dosage) and oxcarbazepine (300 mg daily dosage). These two cases were responsive to these AEDs together with the other two cases responsive to AEDs where daily drug dosages were not mentioned in the reports. A combination of levetiracetam and lamotrigine and a combination of phenytoin, levetiracetam, and folinic acid were used in the other two cases respectively. Though small in proportion these combined data of four cases demonstrated that patients with DS exposed to AED appeared to have seizure freedom in patients with DS.

Among 12 cases with $\mathrm{HH} 50 \%$ underwent surgical intervention consisting of disconnective surgery for 4 cases, resective surgery for 1 case, and radiosurgery for 1 case. Two cases $(17 \%)$ were mentioned for the surgical candidates. One case was not designated as a surgical candidate. In two cases with HH surgical treatment was not mentioned. Two cases received ablative radiotherapy with $\mathrm{I}_{125}$ of which one case underwent $\mathrm{HH}$ radiosurgery (Table 2).

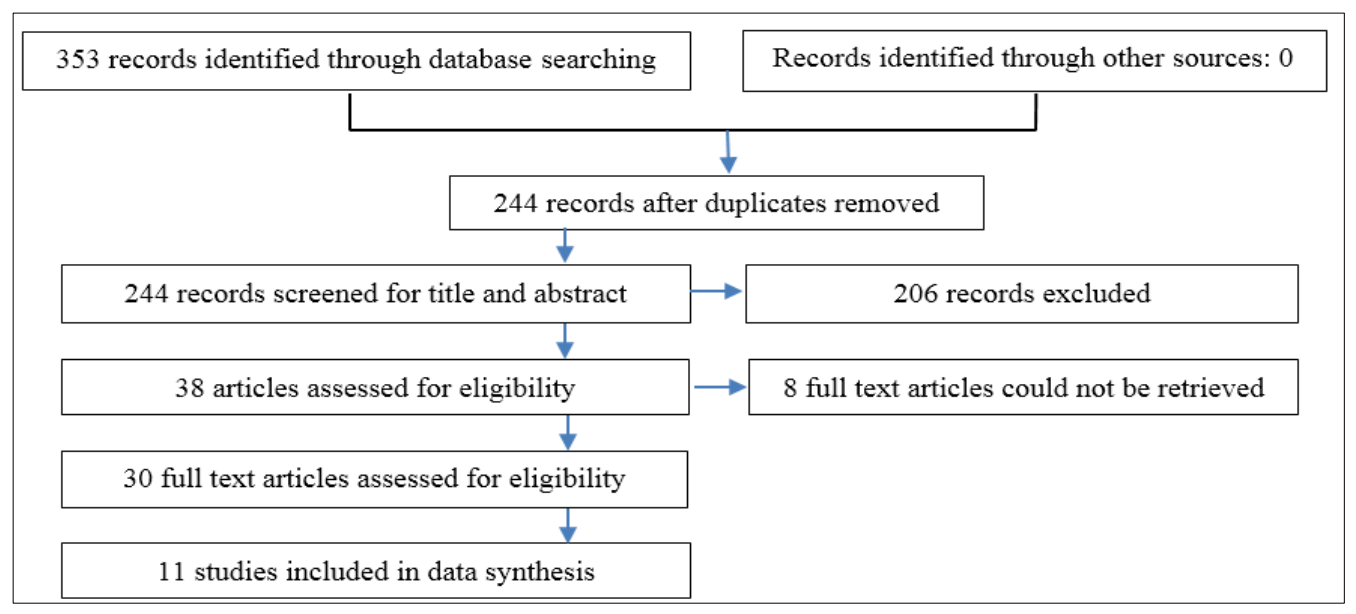

Figure 1: Flow diagram of the study selection process.

Diagrammatic representation of study selection flow conducted according to the PRISMA statement 
Table 1: The demographic characteristics of study cohort.

\begin{tabular}{|llll|}
\hline Variables & All cases & DS alone cases & DS+GS cases \\
\hline Patients, $\mathbf{N}(\boldsymbol{\%})$ & 21 & $7(33.33)$ & $14(66.67)$ \\
\hline Male: female ratio (\%) & $11: 10(52.4: 47.6)$ & $3: 4(42.8: 57.2)$ & $8: 6(57.1: 42.9)$ \\
\hline Age at data acquisition in years (mean+SD) & $6.25 \pm 6.59$ & $8.2 \pm 7.79^{*}$ & $5.28 \pm 5.98^{* *}$ \\
\hline Age of seizure onset in years (mean \pm SD) & $3.2 \pm 3.26$ & $3.1 \pm 3.16$ & $3.25 \pm 3.43^{* * * *}$ \\
\hline
\end{tabular}

*Age of data acquisition was not mentioned in 2 cases, so ages of seizure onset included here; **age of data acquisition was not mentioned in 9 cases, so ages of seizure onset included here; ***age of seizure onset was not mentioned in one case, so age of data acquisition included here
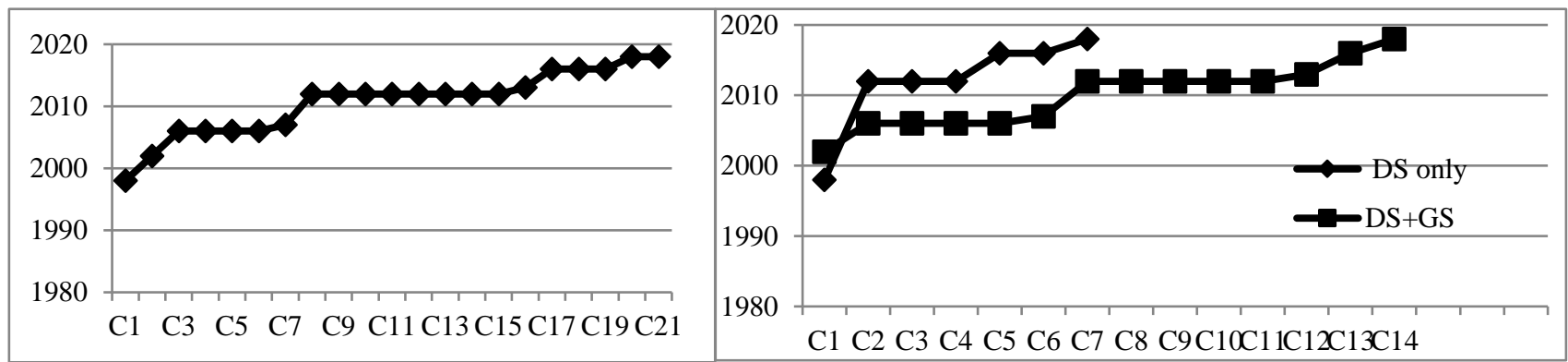

Figure 2: (a) Year wise chronology of all 21 reported cases published in 11 selected articles, and (b) category wise chronology of all 21 reported cases published in 11 selected articles. There are two categories. In DS alone category there are 7 cases and in combined DS and GS category there are 14 cases. DS: dacrystic seizure, GS: gelastic seizure.

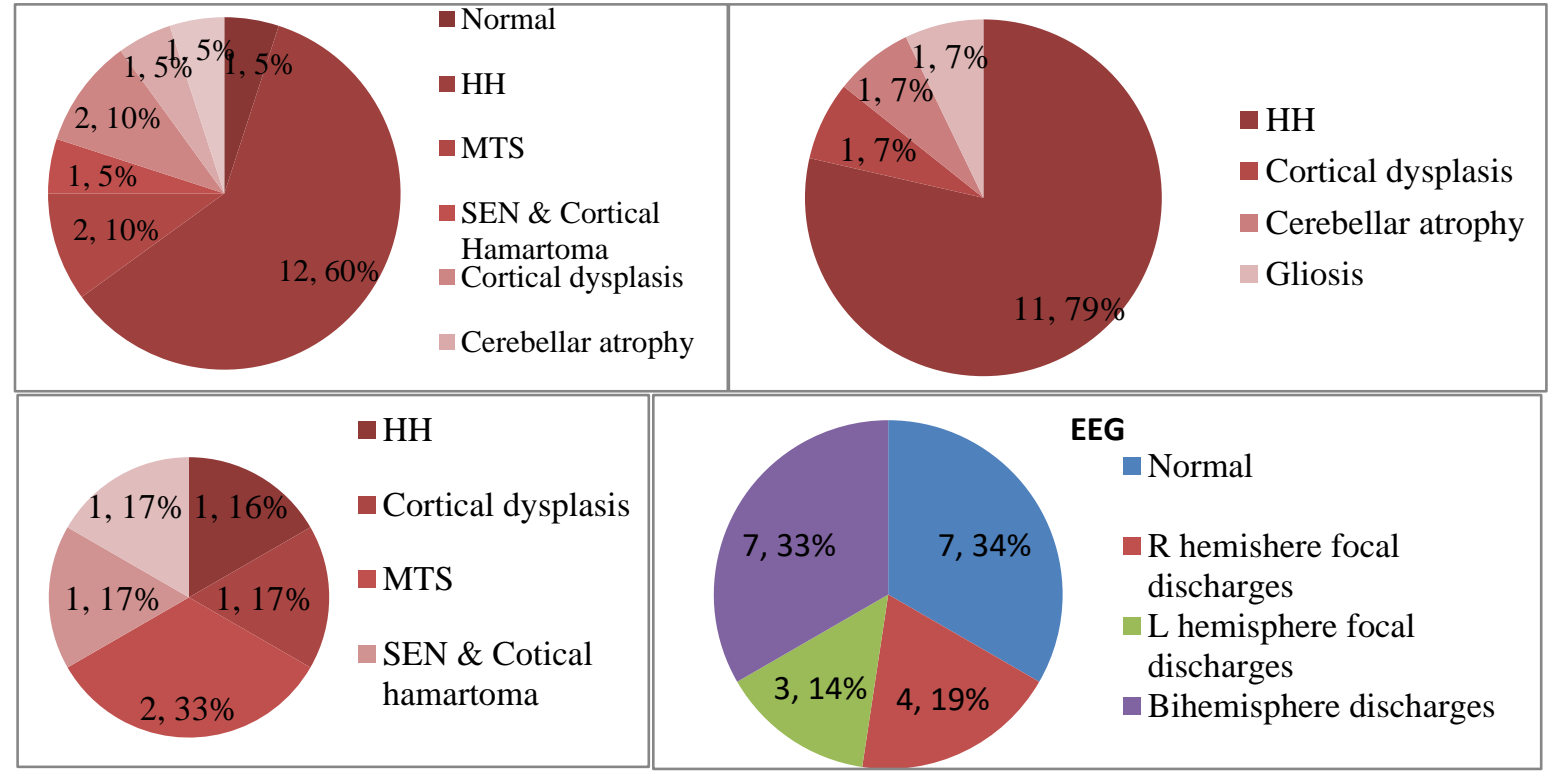

Figure 3: Distribution of findings of neuroimaging and EEG (a) distribution of neuroimaging findings in 20 cases.

Neuroimaging in one case was not mentioned, (b) distribution of neuroimaging findings in all 14 cases with combination of DS and GS, (c) distribution of neuroimaging findings in 6 cases out of total 7 cases DS alone, and (d) distribution of EEG findings in all 21 cases.

DS: dacrystic seizure, GS: gelastic seizure, EEG: electroencephalography

Table 2: Baseline findings of the study cohort.

\begin{tabular}{|ll|}
\hline Variables & Characteristics \\
\hline Imaging-MRI or CT result, $\mathrm{n}=20(\%):$ normal & $1(5.00)$ \\
\hline Abnormal* & $19(95.00)$ \\
\hline Imaging not done $(\%)$ & $1(4.76)$ \\
\hline Interictal EEG findings, $\mathrm{n}=21(\%)$ : normal & $7(33.33)$ \\
\hline
\end{tabular}




\begin{tabular}{|ll|}
\hline Variables & Characteristics \\
\hline Abnormal & $15(66.67)$ \\
\hline Failed to retrieve & $0(0)$ \\
\hline AED treatment, $\mathbf{n = 1 8}(\boldsymbol{\%})$ & $4(22.00)$ \\
\hline AED responsive & $14(78.00)$ \\
\hline Difficult to seizure freedom & $3(14.00)$ \\
\hline AED treatment not mentioned & \\
\hline HH (n=12) & $6(50)$ \\
\hline Resection mentioned $(\%)$ & $2(16.67)$ \\
\hline Surgical candidate $(\%)$ & $1(8.33)$ \\
\hline Not surgical candidate $(\%)$ & $2(16.67)$ \\
\hline Surgical treatment not mentioned $(\%)$ & $2(16.67)$ \\
\hline Radiotherapy with $\mathrm{I}_{125}(\%)^{*}$ & \\
\hline
\end{tabular}

*One case underwent radiosurgery included in resection mentioned

\section{DISCUSSION}

Under the umbrella of the focal onset seizure, DS is a focal emotional seizure with crying which is one type of epileptic 'aura'. ${ }^{19}$ DS is often underdiagnosed and is still not well understood. ${ }^{10,15,20,21}$ It is worthy to mention here that there was more than a four-year treatment gap in a DS case because of unusual semiology mimicking crying spells as the behaviour of a demanding child for which child care professionals were reluctant and underestimated crying as an ictal manifestation. No doubt DS is quite uncommon and unfamiliar among professionals. These significant barriers remain regarding access to diagnosis for DS. Therefore, the likelihood to be misdiagnosed values may be quite larger. In this context, the child care experts should turn out to be alert to the characteristic semiology of DS.

It is well evidenced in this meta-analysis that there are no age thresholds in DS manifestation in children and adolescence. Even in neonates, DS can occur. Considering sex ratio there is a slight male predisposition and categorically there are very negligible variations in sex ratio.

The etiological differences were identified in the comparison of clinical manifestations of DS. Despite limitations, the accumulated data showed most of the cases were associated with hypothalamic hamartomas when the patients manifested a combination of DS and GS. In contrast, when the patients manifested DS alone without GS the underlying lesions were variegated. This metaanalysis demonstrated that lesional dacrystic epilepsy is predominant among children and adolescence. No structural brain lesion demonstrated in one case with computed tomography dictating DS can also occur in patients with normal brain imaging. ${ }^{17}$

The impact of epileptiform discharges in the EEG is challenging for the diagnosis of epilepsy by itself. However, they provide important circumstantial evidence for the diagnosis of epilepsy in the appropriate clinical setting. Considering the sensitivity of routine EEG is around $50 \%$ for the initial EEG, a normal routine EEG finding in this study is $34 \%$ of cases dictating expected sensitivity. ${ }^{22}$ This result does not exclude a diagnosis of dacrystic epilepsy in the setting of convincing clinical evidence. Abnormal EEG finding is supportive for epilepsy and consistent with the structural abnormalities in most of the cases.

Considering the structural brain lesions most of the cases in this meta-analysis are pharmaco-resistant. Only four out of 18 patients in this meta-analysis achieve seizure freedom with antiepileptic drugs. ${ }^{10.15,17,18}$ Though small in proportion this result dictates that seizure freedom is possible in DS cases with appropriate antiseizure medications. However, cases with difficulty to treat with antiepileptic drugs are predominant.

Accompanying of GS dictates the association of HH in DS cases and consequently, DS becomes pharmaco-resistant and needs surgical intervention. However, there is no guaranteed improvement even after epilepsy surgery. Of note, the HH surgery is responsive for gelastic seizures, but crying seizures remain relatively stereotyped.

Being a meta-analysis study focusing on pediatric DS, the author is constrained by the small sample size. In addition, we were not able to include the current manuscripts published in the non-English language. Thus, it is plausible to suppose that some important pediatric case reports were not included in our meta-analysis.

\section{CONCLUSION}

In conclusion, not all crying spells in children are benign; some of them may be ictal crying (DS) which is treatable. So, child care professionals should be aware of this. Further large-scale studies and case reports may have paramount importance to clarify the spectrum of DS in children. Lesional dacrystic epilepsy is predominant and pharmaco-resistant in children. However, antiepileptic drugs lead to achieving seizure remission in few cases. However, there are no guaranteed improvements even after surgical intervention. Of note, the HH surgery is responsive for gelastic seizures, but crying seizures remain 
relatively stereotyped. Though small in proportion, AEDs lead to achieving seizure remission.

Funding: No funding sources

Conflict of interest: None declared

Ethical approval: Not required

\section{REFERENCES}

1. Parvizi J, Anderson SW, Martin CO, Damasio H, Damasio AR. Pathological laughter and crying: a link to the cerebellum. Brain. 2001;124:1708-19.

2. Parvizi J, Coburn KL, Shillcutt SD, Coffey CD, Lauterbach EC, Mendez MF. Neuroanatomy of Pathological Laughing and Crying: A Report of the American Neuropsychiatric Association Committee on Research. J Neuropsychiatry Clin Neurosci. 2009;21:75-87.

3. Blumberg J, Fernandez IS, Vendrame M. Dacrystic seizures: demographic, semiologic, and etiologic insights from a multicenter study in long-term videoEEG monitoring units. Epilepsia. 2012;53:1810-9.

4. Akbar APA, Dale W, Michael SR. Ictal crying. Epilepsy \& Behavior. 2016;59:1-3.

5. Ziligien H. Rire et pleurer spasmodique. Revue Medicale de l'Est. 1906;38:1-12.

6. Offen ML, Davidoff RA, Troost BT, Richey ET. Dacrystic epilepsy. J Neurol Neurosurg Psychiatry. 1976;39:829-34.

7. WHO: Epilepsy facts sheet. World Health Organization, Geneva. 2019. Available at: https://www.who.int/news-room/factsheets/detail/epilepsy. Accessed on 24 June 2021.

8. Luciano D, Devinsky O, Perrine K. Crying seizures. Neurology. 1993;43:2113-7.

9. Moher D, Liberati A, Tetzlaff J, Altman DG PRISMA Group. Preferred reporting items for systematic reviews and meta-analyses: the PRISMA statement. J Clin Epidemiol. 2009;62:1006-12.

10. Dan B, Boyd SG. Dacrystic seizures reconsidered. Neuropediatrics. 1998;29:326-7.

11. Wakai S, Nikaido $\mathrm{K}$, Nihira $\mathrm{H}$, Kawamoto $\mathrm{Y}$, Hayasaka H. Gelastic seizure with hypothalamic hamartoma: proton magnetic resonance spectrometry and ictal electroencephalographic findings in a 4year-old girl. J Child Neurol. 2002;17:44-6.
12. Procaccini E, Dorfmuller G, Fohlen M, Bulteau C, Delalande O. Surgical management of hypothalamic hamartomas with epilepsy: the stereoendoscopic approach. Neurosurgery. 2006;59:336-44.

13. Lopez-Laso E, Mateos Gonzalez ME, Camino Leon R, Jimenez Gonzalez MD, Esparza Rodriguez J. Giant hypothalamic hamartoma and dacrystic seizures. Epileptic Disord. 2007;9:90-3.

14. Mondal K, Sharma S, Aneja S, Kumar A. Unexplained Crying Spells in a Young Child. Indian J Pediatr. 2012;79(1):132-3.

15. Verma R, Praharaj HN. Reflex gelastic-dacrystic seizures following hypoxic-ischaemic encephalopathy. BMJ Case Rep. 2013.

16. Moise AM, Leary L, Morgan LC, Papanastassiou AM, Ákos Szabó C. Ictal laughter and crying: Should they be classified as automatisms? Epilepsy Behav Case Rep. 2016;7:31-3.

17. Raybarman, C. Dacrystic Epilepsy. Indian J Pediatr. 2018;85:563-4.

18. Vidaurre J, Nunley S. Atypical Presentation of a Progressive and Treatable Encephalopathy in an Older Child With Gelastic and Dacrystic Seizures. Semin Pediatr Neurol. 2018;26:95-100.

19. Fisher RS, Cross JH, D'Souza C, French JA, Haut $\mathrm{SR}$, Higurashi $\mathrm{N}$, et al. Instruction manual for the ILAE 2017 operational classification of seizure types. Epilepsia. 2017;58(4):531-42.

20. Burghardt T,Basha MM, Fuerst D, Mittal S. Crying with sorrow evoked by electrocortical stimulation. Epileptic Disord. 2013;15:72-5.

21. Tatum WO, Loddenkemper T. Crying with left temporal lobe seizures and Wada testing. Epilepsy Behav. 2010;18:303-5.

22. Benbadis SR, Beniczky S, Bertram E, MacIver S, Moshé SL. The role of EEG in patients with suspected epilepsy. Epileptic Disord. 2020;22(2):143-55.

Cite this article as: Raybarman C. A meta-analysis of case-reports and case-series of dacrystic epilepsy in children and adolescence. Int J Contemp Pediatr 2021;8:1873-8. 